\title{
Photoproduction and Decay of Light Mesons in CLAS
}

\author{
Moskov Amaryan* \\ Old Dominion University, Norfolk, VA 23508, USA \\ E-mail: mamaryan@odu.edu \\ On behalf of the CLAS Collaboration
}

\begin{abstract}
We present preliminary experimental results on photoproduction and decay of light mesons measured with CLAS setup at JLAB . This include Dalitz decay of pseudoscalar and vector mesons, radiative decay of pseudoscalar mesons as well hadronic decays of pseudoscalar and vector mesons. The collected high statistics in some of decay channels exceeds the world data by an order of magnitude and some other decay modes are observed for the first time. It is shown how the CLAS data will improve the world data on transition form factors of light mesons, Dalitz plot analyses, branching ratios of rare decay modes and other fundamental properties potentially accessible through the light meson decays.
\end{abstract}

The 7th International Workshop on Chiral Dynamics,

August 6 -10, 2012

Jefferson Lab, Newport News, Virginia, USA

\footnotetext{
* Speaker.
} 


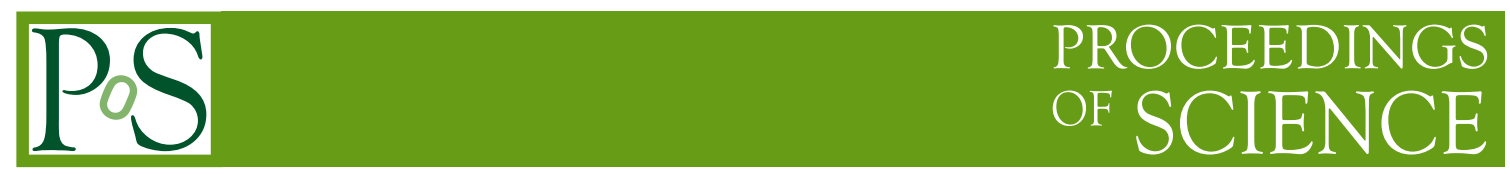

\section{Introduction}

Low energy Quantum Chromodynamics (QCD) is responsible for the binding of hadrons and for the mass of the visible universe. A unique way to explore low energy QCD is by measuring the decays of light mesons, specifically the $\pi^{0}, \eta$ and $\eta^{\prime}$ pseudoscalar mesons and light vector mesons $\rho, \omega, \phi$. In particular, the $\eta$ and $\eta^{\prime}$ mesons present important information on the low energy dynamics of QCD: the mechanism of spontaneous chiral symmetry breaking and the $U_{A}(1)$ anomaly. The importance of this topic is shown by the number of experiments performed at an impressive array of facilities including KLOE, CLEO, BES, MAMI, Bonn, COSY, BABAR, BELLE, and CERN. The CLAS photoproduction data has superior statistics in many channels, exceeding that of published results by up to an order of magnitude.

\section{Dalitz decays}

The branching ratios for radiative decay of pseudoscalar mesons $\pi^{0}$ and $\eta$ have been measured and are recorded by the PDG [1], however there is only an upper limit quoted for $\eta^{\prime} \rightarrow e^{+} e^{-} \gamma$.

In this proposal we briefly present our preliminary distribution of the $e^{+} e^{-} \gamma$ invariant mass from CLAS photoproduction data. This is a $\mathrm{H}\left(\gamma, p e^{+} e^{-} \gamma\right) \mathrm{X}$ four-fold coincidence event sample with an upper bound on the missing energy.

Peaks of $\pi^{0}, \eta$ and $\eta^{\prime}$ are shown separately, with fitted positions corresponding to their PDG values. In addition, there is a clear signal in the $\rho-\omega$ region, and a small peak at the $\phi$-mass. With a branching ratio of $(1.174 \pm 0.035) \%$, the three body decay $\pi^{0} \rightarrow e^{+} e^{-} \gamma$ is the second most important decay channel of the neutral pion and is deeply connected to the main decay mode $\pi^{0} \rightarrow \gamma \gamma(B r=98.823 \pm 0.034 \%)$ with anomalous $\pi^{0} \gamma \gamma$ vertex. Significant interest to the Dalitz decay of $\pi^{0}$ lies in the fact that it provides information on the semi off-shell $\pi^{0} \rightarrow \gamma \gamma^{*}$ transition form factor $F_{\pi^{0} \gamma \gamma^{*}}\left(q^{2}\right)$ in the time-like region, and more specifically on its slope parameter $a_{\pi}$. The determinations of $a_{\pi}$ obtained from the differential decay rate of Dalitz decay $a_{\pi}=-0.11 \pm 0.03 \pm$ 0.08 [2], $a_{\pi}=+0.026 \pm 0.024 \pm 0.0048$ [3], $a_{\pi}=+0.025 \pm 0.014 \pm 0.026$ [4]. Here $a_{\pi}$ is defined from the following expression for the decay rate [5]

$$
\begin{array}{r}
\frac{d \Gamma\left(\pi^{0} \rightarrow e^{+} e^{-} \gamma\right)}{d x \Gamma\left(\pi^{0} \rightarrow \gamma \gamma\right)}=\left(\frac{d \Gamma}{d x}\right)_{Q E D} \times|F(x)|^{2} \\
\left(\frac{d \Gamma}{d x}\right)_{Q E D}=\frac{2 \alpha}{3 \pi} \frac{1}{x}(1-x)^{3}\left(1+\frac{r}{2 x}\right)\left(1-\frac{r}{x}\right)^{1 / 2} \\
F(x)=1+a_{\pi} x
\end{array}
$$

where $x=m_{e^{+} e^{-}}^{2} / m_{\pi^{0}}^{2}, r=4 m_{e}^{2} / m_{\pi^{0}}^{2}$, and $F(x)$ is $\pi^{0}$ transition form factor.

These measurements have large error bars, as compared to the values extracted from the extrapolation of data at higher energies in the space-like region, $Q^{2}=-q^{2}>0.5 \mathrm{GeV}^{2}$, obtained by CELL0 and CLEO collaborations: $a_{\pi}=+0.0326 \pm 0.0026 \pm 0.0026[6], a_{\pi}=+0.0303 \pm 0.0008 \pm$ $0.0009 \pm 0.0012$ [7]. Extraction of $a_{\pi}$ from experimental data obtained by CELLO [6] , CLEO [7] and BABAR [8] experiments is model dependent. A direct and accurate determination of $a_{\pi}$ from the decay $\pi^{0} \rightarrow e^{+} e^{-} \gamma$ would offer very important source of information to understand transition 
form factor of neutral pion. Another reason for the importance of this information is related to the precise determination of the most uncertain light-by-light radiative corrections to the anomalous magnetic moment of the muon, $a_{\mu}$, measured in g-2 experiment [9].

In Fig. 1 we show peaks of $\pi^{0}, \eta$ and $\eta^{\prime}$ in the invariant mass $M\left(e^{+} e^{-} \gamma\right)$ fitted with a Gaussian plus second order polynomial function. As one can see we have very clean signal of $\pi^{0}$ and $\eta$ mesons. The $\eta^{\prime}$ peak is reconstructed in the Dalitz decay mode for the first time.
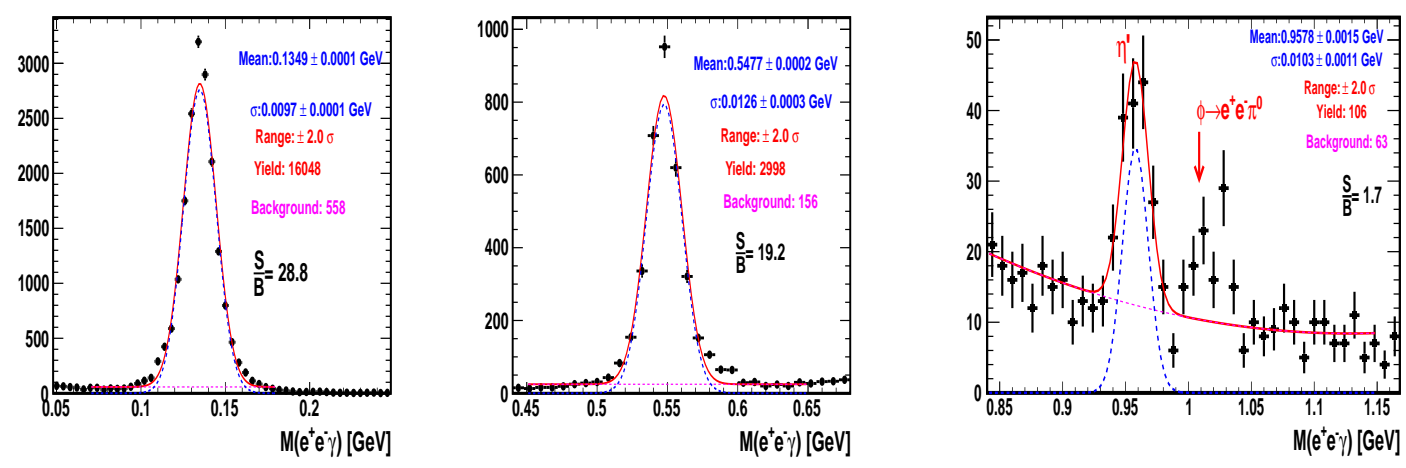

Figure 1: Invariant mass, $M\left(e^{+} e^{-} \gamma\right)$, distribution in different mass regions. Left panel: peak of $\pi^{0}$, middle panel: peak of $\eta$. The right panel shows peak of $\eta^{\prime}$ and partially reconstructed peak of $\phi$ meson $e^{+} e^{-} \pi^{0}$ decay mode.

New experiment is proposed in KLOE-2 [10] to measure $F_{\pi^{0}}\left(Q^{2}, 0\right)$ with statistical precision shown in Fig. 2 (left panel). Statistically significant data are already collected with CLAS. The CLAS g12 raw data under the $\pi^{0}$ peak in $e^{+} e^{-} \gamma$ decay mode are presented in Fig. 2 (right panel), which will allow to extract the slope of $Q^{2}$ dependence, $a_{\pi^{0}}$, in the time-like region at very low $Q^{2}$ with statistical accuracy for the first time comparable or better than that extracted from $e^{+} e^{-} \rightarrow \pi^{0}$ data at higher $Q^{2}$ in space-like region.
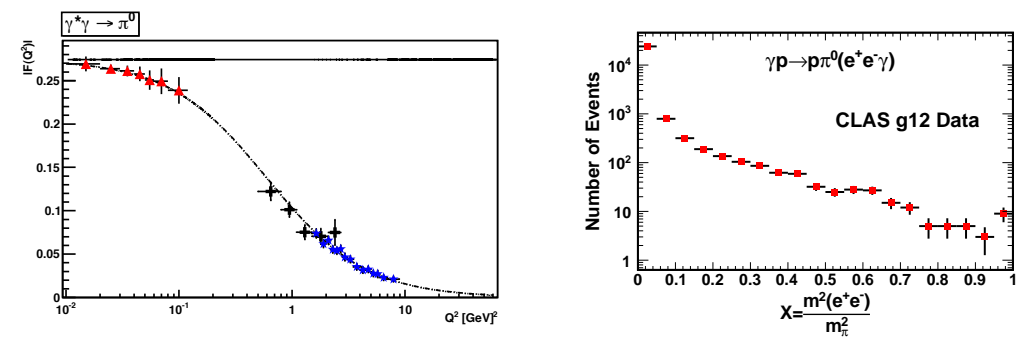

Figure 2: Left panel: experimental data from CELLO (black crosses) and CLEO (blue stars) at low $Q^{2}$ together with KLOE-2 proposal (red triangles) to measure $F_{\pi^{0}}\left(Q^{2}, 0\right)$ at very low $Q^{2}$ [10]. Dashed line is the $F\left(Q^{2}\right)$ form factor according to LMD+V model [11], solid line is $F(0)$ given by Wess-Zumino-Witten term. Right panel: raw data from CLAS g12 experiment within $2 \sigma$ of the peak of $\pi^{0}$ from Fig. 1 (left panel), plotted vs the ratio $x=m_{e^{+} e^{-}}^{2} / m_{\pi^{0}}^{2}$

In Fig. 3 left panel we present world data on $\eta$ form factor, on the right panel we present 
projected form factor with statistical errors obtained from CLAS g12 experiment within $2 \sigma$ of the peak of $\eta$. This CLAS dataset for the $\eta$ Dalitz decay exceeds by several times the statistics of the recent MAMI CB/TAPS [12], and NA60 dimuon $\left(\eta \rightarrow \mu^{+} \mu^{-} \gamma\right)$ [13] measurements. Both of these results observe a clear deviation of the form factor from the simple QED result based on the $\eta \rightarrow \gamma \gamma$ matrix element. The CLAS data can significantly improve the extraction of the form factor over the full accessible range $0<m_{e^{+}} e^{-} \leq m_{\eta}$, including the region below twice the muon mass, which is inaccessible in the NA60 experiment.
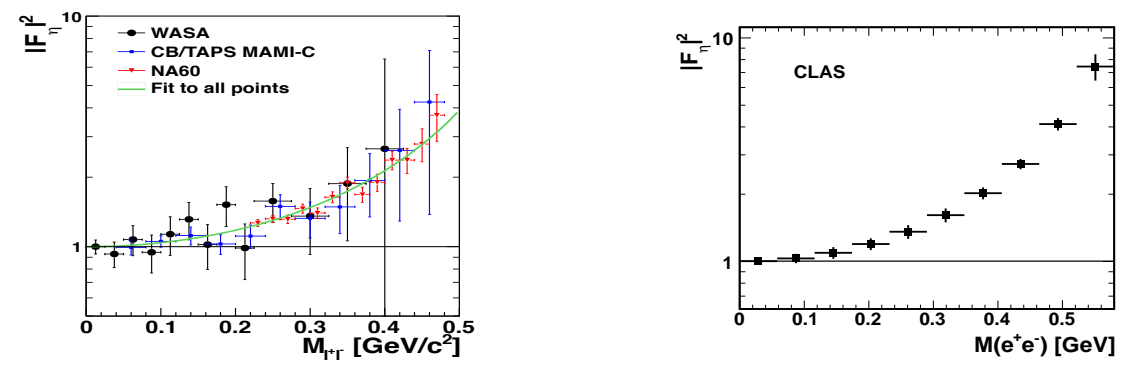

Figure 3: Left panel: Experimental spectrum of the squared transition form factor, $\left|F_{\eta}\right|^{2}$, as a function of $M\left(l^{+} l^{-}\right)$. The green, solid line is the fit to all experimental points. The black, solid line is the QED model assumption of a point-like meson. Right panel: projected form factor with statistical errors obtained from CLAS g12 experiment within $2 \sigma$ of the peak of $\eta$ from Fig. 1 (middle panel), plotted vs $M\left(e^{+} e^{-}\right)$.

In the Dalitz decays of vector mesons, the vector meson decays into a pseudoscalar meson and a lepton pair, formed by internal conversion of an intermediate virtual photon. A theoretical explanation for the observed deviation from VMD is in need of improved experimental input and is pursued with current theoretical efforts [14] going beyond VMD in a systematic way. It is important to determine the transition form factors for vector mesons via Dalitz decays with different experimental methods. This measurement will have the highest statistics achieved so far.
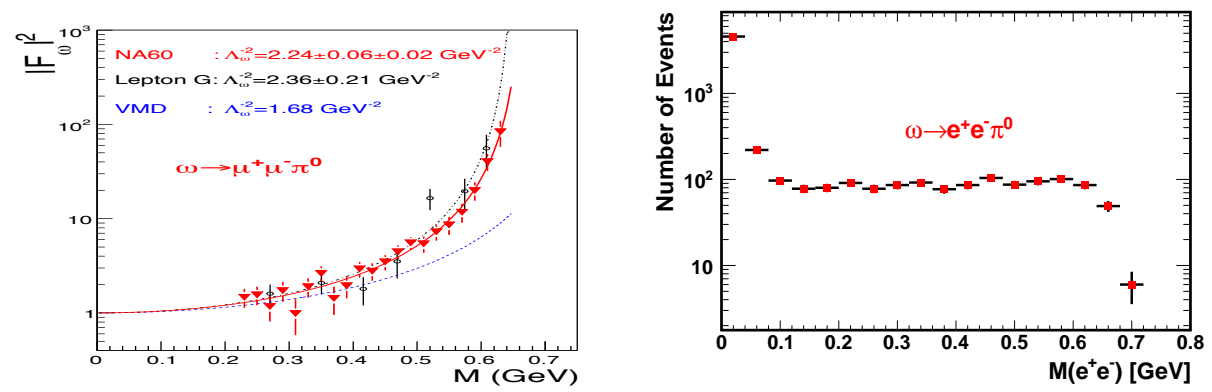

Figure 4: Left panel: transition form factor of $\omega$ from NA60 experiment [13] (red triangles), compared to the previous measurement by the Lepton-G experiment [15] (open circles) and to the expectation from VMD (blue dashed line). The solid red and black dashed-dotted lines are results of fitting the experimental data with the pole dependence $\left|F^{2}\right|=\left(1-M^{2} / \Lambda^{2}\right)^{-2}$. Right panel number of events from the CLAS g12 data are plotted as a function of invariant mass of $e^{+} e^{-}$pairs.

In the left panel in Fig. 4 the transition form factor of $\omega$ meson is extracted from the NA60 
experiment [13] in the $\omega \rightarrow \mu^{+} \mu^{-} \pi^{0}$ decay mode as a function of invariant mass of $\mu^{+} \mu^{-}$pairs. In the right panel number of events from from $\omega \rightarrow e^{+} e^{-} \pi^{0}$ decay obtained from the CLAS g12 data are presented as a function of invariant mass of $e^{+} e^{-}$pairs.

\section{Radiative and Hadronic Decays}

In addition to the Dalitz decays of light mesons huge statistics is collected with the CLAS setup on radiative $\eta, \eta^{\prime} \rightarrow \pi^{+} \pi^{-} \gamma$ and hadronic decays $\eta, \omega, \eta^{\prime} \rightarrow \pi^{+} \pi^{-} \pi^{0}, \eta^{\prime} \rightarrow \pi^{+} \pi^{-} \eta$ [16]. The Dalitz plot analysis of these decay channels will be carried out with highest statistical precision achieved so far.

\section{Summary}

In conclusion, from our preliminary analyses one can see that the CLAS data on photoproduction and decay of light mesons can contribute significantly to essential topics of low energy QCD. The data already on tape at JLab in some of these channels have statistics that is not in a reach of other world facilities.

\section{References}

[1] K. Nakamura et al., "Review of particle physics," J. Phys., vol. G37, p. 075021, 2010.

[2] H. Fonvieille, N. Bensayah, J. Berthot, P. Bertin, M. Crouau, et al., "Dalitz decay: $\pi^{0} \rightarrow e^{+} e^{-} \gamma$ and the $\pi^{0}$ electromagnetic transition form-factor," Phys.Lett., vol. B233, pp. 65-68, 1989.

[3] F. Farzanpay, P. Gumplinger, A. Stetz, J. Poutissou, I. Blevis, et al., "Measurement of the slope of the $\pi^{0}$ electromagnetic form-factor," Phys.Lett., vol. B278, pp. 413-418, 1992.

[4] R. Meijer Drees et al., "Measurement of the pi0 electromagnetic transition form-factor," Phys.Rev., vol. D45, pp. 1439-1447, 1992.

[5] L. Landsberg, "Electromagnetic Decays of Light Mesons," Phys.Rept., vol. 128, pp. 301-376, 1985.

[6] H. Behrend et al., "A Measurement of the pi0, eta and eta-prime electromagnetic form-factors," Z.Phys., vol. C49, pp. 401-410, 1991.

[7] J. Gronberg et al., "Measurements of the meson - photon transition form-factors of light pseudoscalar mesons at large momentum transfer," Phys.Rev., vol. D57, pp. 33-54, 1998.

[8] B. Aubert et al., "Measurement of the $\gamma \gamma^{*} \rightarrow \pi^{0}$ transition form factor," Phys.Rev., vol. D80, p. $052002,2009$.

[9] H. N. Brown et al., "Precise measurement of the positive muon anomalous magnetic moment," Phys. Rev. Lett., vol. 86, pp. 2227-2231, Mar 2001.

[10] H. Czyz, S. Ivashyn, A. Korchin, and O. Shekhovtsova, "Two-photon form factors of the $\pi^{0}, \eta$ and $\eta^{\prime}$ mesons in the chiral theory with resonances," Phys.Rev., vol. D85, p. 094010, 2012.

[11] D. Babusci, H. Czyz, F. Gonnella, S. Ivashyn, M. Mascolo, et al., "On the possibility to measure the $\pi^{0} \rightarrow \gamma \gamma$ decay width and the $\gamma^{*} \gamma \rightarrow \pi^{0}$ transition form factor with the KLOE-2 experiment,"

Eur.Phys.J., vol. C72, p. 1917, 2012. 
[12] H. Berghauser, V. Metag, A. Starostin, P. Aguar-Bartolome, L. Akasoy, et al., "Determination of the eta-transition form factor in the $\gamma p \rightarrow p \eta \rightarrow p \gamma e^{+} e^{-}$reaction," Phys.Lett., vol. B701, pp. 562-567, 2011.

[13] R. Arnaldi et al., "Study of the electromagnetic transition form-factors in $\eta \rightarrow \mu^{+} \mu^{-} \gamma$ and $\omega \rightarrow \mu^{+} \mu^{-} \pi^{0}$ decays with NA60," Phys.Lett., vol. B677, pp. 260-266, 2009.

[14] C. Terschlusen and S. Leupold, "Electromagnetic transition form factors of light vector mesons," Phys.Lett., vol. B691, pp. 191-201, 2010.

[15] R. Dzhelyadin, S. Golovkin, A. Konstantinov, V. Konstantinov, V. Kubarovsky, et al., "Study of the electromagnetic transition form-factor in $\omega \rightarrow \pi^{0} \mu^{+} \mu^{-}$decay," Phys.Lett., vol. B102, p. 296, 1981.

[16] P. Adlarson, M. Amaryan, M. Bashkanov, F. Bergmann, M. Berlowski, et al., "Proceedings of the second International PrimeNet Workshop," arXiv:1204.5509. 